\title{
Release of Full-Length EphB2 Receptors from Hippocampal Neurons to Cocultured Glial Cells
}

\author{
Jenny Lauterbach and Rüdiger Klein \\ Max-Planck Institute of Neurobiology, D-82152 Martinsried, Germany
}

\begin{abstract}
Glial cells are known to actively participate in neuronal development by shaping neuronal connections through axon pruning and by controlling dendritic spine morphology. These functions may in part be mediated by engulfment of neuronal structures and transendocytosis of neuronal material into glial cells. These processes are not well understood, and the molecular components that mediate these events have primarily been elusive. Here, we implicate the Eph/ephrin signaling system in trans-endocytosis events at the neuronto-glia interface. Using time-lapse microscopy, we show that hippocampal neurons exogenously expressing EphB2 receptors release or pinch-off EphB2-containing vesicles at sites of neuron-to-glia contact. Cocultured glial cells endogenously express the corresponding ephrinB ligands and are able to trans-endocytose full-length EphB2 from neighboring cells. Although Eph/ephrin signaling often occurs in a bidirectional manner, the observed vesicle release from neurons to glia was only observed in a unidirectional manner, i.e., when the neurons expressed EphB2, but not ephrinBs. These findings suggest that Eph/ephrin signaling is involved in the glial cell-mediated fine sculpting of neuronal structures.
\end{abstract}

Key words: astrocyte; endocytosis; Eph; ephrin; hippocampal neuron; receptor; tyrosine kinase

\section{Introduction}

Traditionally, glial cells have been viewed to function as housekeeping cells and as scavengers that help to remove degenerating axons and neurons (Nedergaard et al., 2003). More recently, astrocytes are being appreciated as dynamic regulators of CNS synaptogenesis and synaptic transmission (Volterra and Meldolesi, 2005). In flies, glial cells engulf degenerating axons during developmentally regulated axon pruning (Awasaki and Ito, 2004). In vertebrates, during synapse elimination at the developing neuromuscular junction, withdrawing axons shed fragments into surrounding glial cells (Bishop et al., 2004). In the brain, astrocytes may also have an active role in developmentally regulated structural remodeling of neuronal processes. In the adult rodent hippocampus, double-membrane structures (spinules) were found through electron microscopy in axons and astrocytes derived from nearby dendritic spines, suggesting exchange of material among neurons and glial cells (Spacek and Harris, 2004). The molecular components that underlie these trans-endocytosis events, however, remained essentially unknown.

Eph receptor tyrosine kinases and their ephrin ligands have important roles in the establishment of neuronal and vascular networks during development and in the regulation of structural

Received June 26, 2006; revised Sept. 22, 2006; accepted Sept. 24, 2006.

This work was in part supported by the Max-Planck Society, the European Union (ENDOTRACK), and Deutsche Forschungsgemeinschaft Grant SPP1172. We thank E. Hoppe and Y. A. Barde for the GFP-TrkB.T1 expression plasmid, A. Kossel, M. Zimmer, C. Eßmann, and B. Götze for valuable comments and experimental help, and L. Dehmelt, M. Sperling, and L. Meyn for experimental help. We acknowledge T. Mäkinen and K. Deininger for critically reading this manuscript.

Correspondence should be addressed to Rüdiger Klein, Max-Planck Institute of Neurobiology, Am Klopferspitz 18, D-82152 Martinsried, Germany. E-mail: rklein@neuro.mpg.de.

J. Köhler's present address: The Scripps Research Institute, 10550 North Torrey Pines Road, San Diego, CA 92037. DOl:10.1523/JNEUROSCI.2697-06.2006

Copyright $\odot 2006$ Society for Neuroscience $\quad 0270-6474 / 06 / 2611575-07 \$ 15.00 / 0$ and physiological functions of excitatory synapses in the adult brain (Palmer and Klein, 2003; Davy and Soriano, 2005; Eichmann et al., 2005; Flanagan, 2006). Because of the nature of the membrane-anchored ephrins, signaling between Ephs and ephrins is bidirectional, consisting of forward signals into the Eph receptor-expressing cell and of reverse signals into the ephrinexpressing cell. Eph receptors are subdivided into A-type and B-type receptors, reflecting their binding affinities to either glycosylphosphatidylinositol-anchored ephrinAs (A1-A5) or transmembrane ephrinBs (B1-B3) (Kullander and Klein, 2002; Pasquale, 2005).

EphrinA3 is found on astrocytic processes near synaptic terminals, and the interaction between ephrinA3 and neuronal EphA4 stabilizes spine morphology in the hippocampus (Murai et al., 2003). In the context of cell-to-cell-mediated repulsion, ephrinB/EphB signaling leads to bidirectional endocytosis of the ephrinB/EphB protein complex (Marston et al., 2003; Zimmer et al., 2003). For these experiments, we used cells expressing EphB2 carrying in their cytoplasmic tail one variant of enhanced green fluorescent protein (GFP). On contact with a cell expressing ephrinB1, EphB2 receptors were rapidly clustered and internalized/ endocytosed into both cells. Stimulation of ephrinB-expressing neuronal growth cones with cells expressing EphB2 caused rapid growth cone collapse and axon withdrawal. When EphB2 was C-terminally truncated and endocytosis into the stimulator cell was blocked, axon withdrawal was delayed. Thus, both cells actively remove the adhesive ephrin/Eph protein complex from the surface to allow repulsion to happen (Marston et al., 2003; Wilkinson, 2003; Zimmer et al., 2003).

Until now, we have shown that neurons can engulf ephrin/ Eph complexes from other cells (Zimmer et al., 2003). It was not known whether neurons could also be the target of "attack" from 


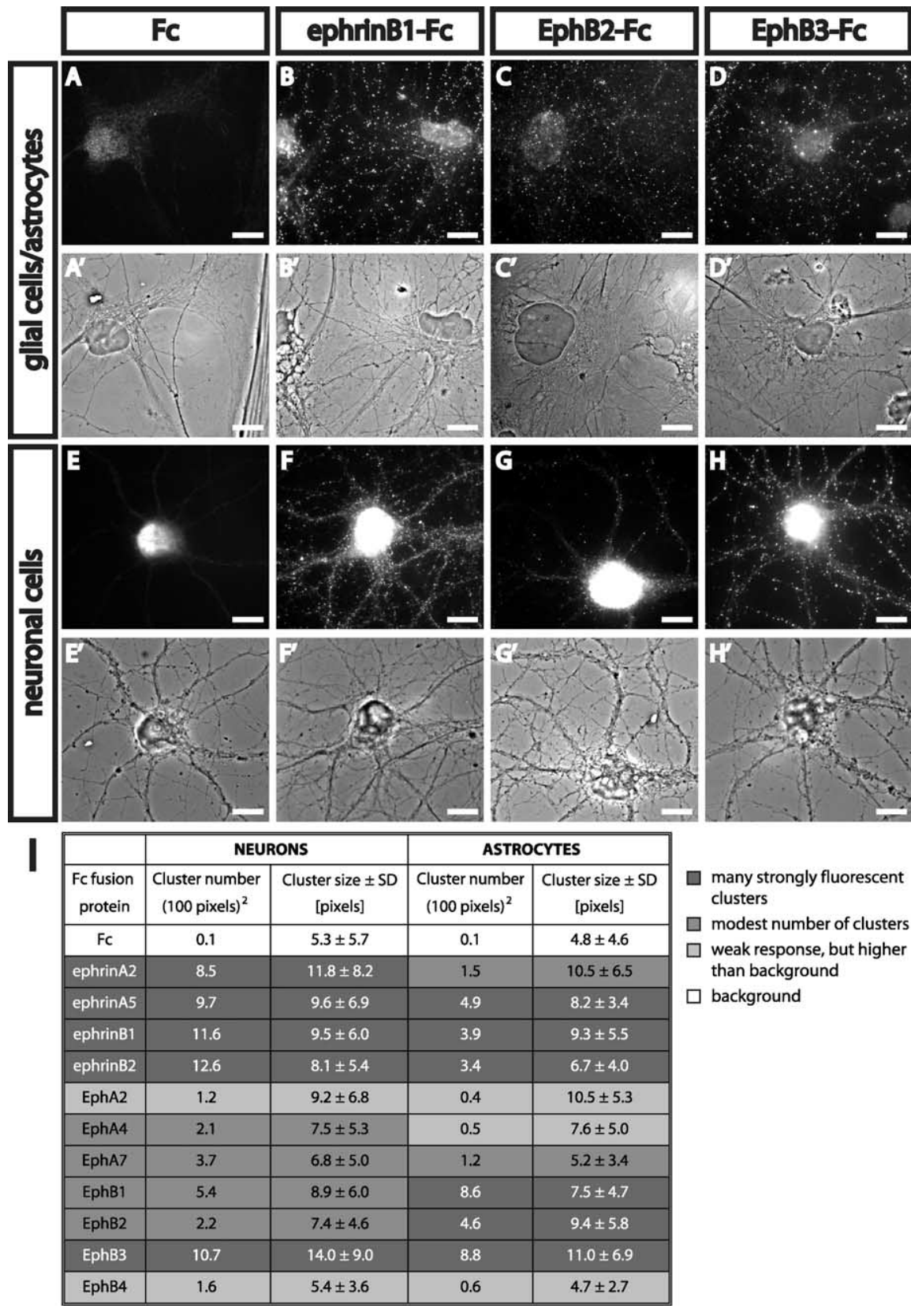

Figure 1. Expression of ephrinBs and EphB receptors in mixed hippocampal cultures. The low-density 14 DIV primary hippocampal cultures were stimulated for $15 \mathrm{~min}$ with the indicated preclustered ectodomain ephrin/Eph-Fc fusion proteins or as a control with preclustered control $\mathrm{Fc}$ before fixation. After permeabilization, the cultures were stained with antibodies against the $\mathrm{Fc}$ portion $(\boldsymbol{A}-\boldsymbol{H})$. Specific binding of the $\mathrm{Fc}$ fusion proteins leads to the appearance of characteristic clusters (as in $\boldsymbol{B}$ ). Glial cells and neurons were identified by their typical non-neuronal like (large cytoplasm, compact morphology; $\boldsymbol{A}^{\prime}-\boldsymbol{D}^{\prime}$ ) and neuronal-like (multiple neurites; $\boldsymbol{E}^{\prime}-\boldsymbol{H}^{\prime}$ ) morphologies by bright-field microscopy, respectively. The non-neuronal/glial-like morphology was correlated with GFAP-positive cells (supplemental Fig. 1, available at www.jneurosci.org as supplemental material). Scale bars, 10 $\mu \mathrm{m}$. I, Quantification of ephrin and Eph expression. Three to four images for each condition were analyzed using MetaMorph software (supplemental Materials and Methods, available at www.jneurosci.org as supplemental material). The background ( $\mathrm{Fc}$ ) staining was subtracted from each image, and only round unmerged clusters were counted.

other cells. Here we show that astrocytes trans-endocytose EphB2 receptors from cocultured hippocampal neurons. Using timelapse microscopy, we demonstrate that hippocampal neurons release or pinch-off EphB2-containing vesicles from the neuron at sites of neuron-to-glia contact.

\section{Materials and Methods}

Expression constructs. The vector, pEYFP-mem, encoding for the membrane-tagged form (mem) of enhanced yellow fluorescent protein
(EYFP) was purchased from Clontech (Mountain View, CA). The GFP-TrkB.T1 plasmid was from E. Hoppe (Merck KGaA, Grafing, Germany) and Y. A. Barde (Biozentrum, University of Basel, Basel, Switzerland) and corresponds to the previously described hemagglutinin (HA)trkB ICD (Bibel et al., 1999), only the $\mathrm{N}$-terminal HA tag was replaced by EGFP (Clontech). The EphB2-YFP construct (pJK12) was described previously (Zimmer et al., 2003). To generate YFP-ephrinB1 (pJK31), the EYFP coding region of pEYFP-N1 (Clontech) was cloned in frame into SalI/BsRGI sites of pJP136 (Bruckner et al., 1999). The generation of the YFP-ephrinB2 expression construct is described in supplemental Materials and Methods (available at www.jneurosci.org as supplemental material).

Neuronal cultures. Hippocampal neurons were dissected from embryonic day 18.5 rat embryos and plated onto coated glass coverslips (Marienfeld, Bad Mergentheim, Germany) or live-cell glass-bottomed dishes with or without grid (high density, P35G-0-14-C; low density, P35G-2-14-CGRD; MatTek, Ashland, MA) [1 $\mathrm{mg} / \mathrm{ml}$ poly-D-lysine (Sigma, St. Louis, MO) and $5 \mu \mathrm{g} / \mathrm{ml}$ laminin (Invitrogen, Carlsbad, CA)] at densities of either 17,000 cells $/ \mathrm{cm}^{2}$ in Neurobasal-B27 medium (Invitrogen) or 5000 cells $/ \mathrm{cm}^{2}$ in DMEM-B27 (Goetze et al., 2003). EphB2-YFP release was blocked by bath application of $1-4 \mu \mathrm{g} / \mathrm{ml}$ unclustered ephrinB1-Fc (constant fragment of immunoglobulin heavy chain) or EphB2-Fc (R \& D Systems, Minneapolis, MN) during live-cell imaging compared with control Fc (Jackson ImmunoResearch, West Grove, PA). Cultures were imaged for 35 min, then Fc proteins were added, and imaging continued for another $65 \mathrm{~min}$. Transendocytosed fluorescent vesicles in $100 \times 100$ pixels ${ }^{2}$ regions were counted $30 \mathrm{~min}$ before and 30 min after Fc protein addition (at the end of the imaging period). Time-lapse imaging is described in supplemental Materials and Methods (available at www.jneurosci.org as supplemental material).

Primary cultures of astrocytes. For astrocytic cultures (de Hoop et al., 1994), cerebral hemispheres were collected, trypsinized, and physically dissociated. Approximately 60,000 cells/ $\mathrm{cm}^{2}$ were plated onto untreated flasks (Nalge Nunc, Rochester, NY) in MEM-horse serum (HS). After cell attachment, the medium was replaced. Glial cultures were split at a 1:3 ratio. For feeder layer preparation of low-density cultures, astrocytes were seeded onto glass coverslips (outer diameter of $10 \mathrm{~mm}$ ) with paraffin dots in MEM-HS and cultured until $\sim 80 \%$ confluence, and then medium was replaced with neuronal MEM-B27. After 3 d, one to two feeder coverslips were flipped over into the live-cell dishes containing 3-5 $\mathrm{d}$ in vitro (DIV) hippocampal cultures, and half of the medium was replaced with the glial conditioned medium.

\section{Results}

Expression of endogenous ephrins and Eph receptors in astrocytes

Astrocytes of the CNS have been shown previously to express selected ephrins and to upregulate Eph/ephrin expression in re- 

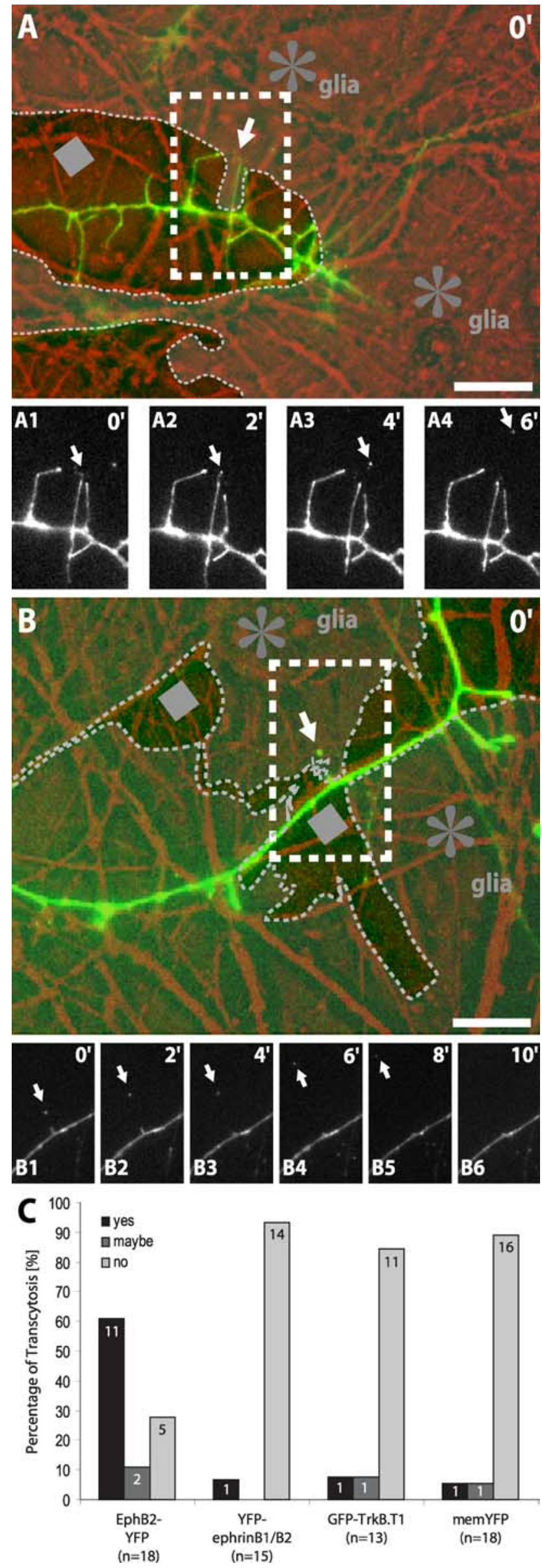

sponse to injury (Conover et al., 2000; Bundesen et al., 2003; Murai et al., 2003; Goldshmit et al., 2004; Wang et al., 2005). Here we assessed the expression of endogenous ephrins and Ephs in mixed primary hippocampal cultures of neurons and astrocytes. Astrocytes were identified by their characteristic morphology and by immunostainings for glial fibrillary acidic protein (GFAP) (supplemental Fig. 1, available at www.jneurosci.org as supplemental material). We used soluble ephrin-Fc and Eph-Fc fusion proteins to stain for their respective binding partners on the surface of cultured cells as described previously (Harbott and Nobes, 2005 and references within). Application of preclustered ephrinB1-Fc, but not of control Fc, to the culture medium elicited many strongly fluorescent clusters on cells with astrocyte morphology, indicating the presence of endogenous EphBs (Fig. $\left.1 A, A^{\prime}, B, B^{\prime}\right)$. Similar responses to ephrinB1-Fc were observed on cocultured neurons (Fig. $1 F, F^{\prime}$ ). A comparison with ephrinB2-Fc revealed similar signal intensities on neurons and astrocytes, suggesting that the endogenous EphB receptors had no binding preference to either ephrinB1 or ephrinB2 (Fig. 1I) (supplemental Fig. $\left.2 B, B^{\prime}\right)$. A marked downregulation of EphB receptors [as reported for EphB2 (Henderson et al., 2001)] was not observed possibly because of the somewhat higher cell density of our cultures. Among the EphB-Fc fusion proteins, EphB1-Fc, EphB2-Fc, and EphB3-Fc elicited similar numbers of clusters on astrocytes, whereas neurons showed strong preference for EphB3-Fc (Fig. 1C,D, G-I) (supplemental Fig. 2, available at www.jneurosci.org as supplemental material). Regarding A-class ephrins, ephrinA2-Fc and ephrinA5-Fc induced similar numbers of clusters on hippocampal neurons, whereas astrocytes showed a stronger response to ephrinA5-Fc compared with ephrinA2-Fc (Fig. 1I) (supplemental Fig. 3, available at www.jneurosci.org as supplemental material). The responses to EphA-Fc proteins were generally weak on both astrocytes and neurons, indicating that the levels of endogenous ephrinAs were low in both cell types (Fig. 1I) (supplemental Fig. 3, available at www.jneurosci.org as supplemental material). Whereas the numbers of clusters varied

\section{$\leftarrow$}

Figure 2. Release of EphB2-containing vesicles from 16-d-old neurons. $A, B$, High-density 14 DIV primary hippocampal cultures were transfected with expression constructs coding for fluorescently tagged EphB2-YFP receptors. Two days later, transfected neurons were imaged by time-lapse microscopy at 1 frame/min. Large images show merge of phase contrast in red and the EphB2-YFP fluorescence of transfected neurites (and its growth cone; $\boldsymbol{A}$ ) in green. Dashed boxes indicate the imaged area in the bottom row. Glial cells (asterisk) are indicated by lighter areas surrounded by dashed lines. Darker areas indicate glia-free area (diamond). Scale

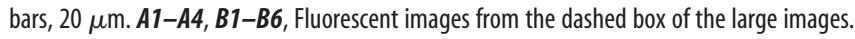
Time points of imaging are indicated in top right corners. A1-A4, Within 6 min, a fluorescent cluster is moving away from the middle filopodium-like protrusion toward a neighboring cell, most likely a glial cell in close contact with the neuron (fluorescent cluster indicated by arrow) (supplemental movie S1, available at www.jneurosci.org as supplemental material). B1-B6, Within $8 \mathrm{~min}$, a fluorescent cluster is moving away from a small neuronal filopodium toward a neighboring cell, most likely a glial cell (fluorescent cluster indicated by arrow) (supplemental movie S2, available at www.jneurosci.org as supplemental material). C, Comparison of efficiency of release of fluorescent clusters generated by different fusion proteins. Primary hippocampal high-density cultures were transfected at 13-15 DIV with expression constructs coding for either EphB2-YFP, YFP- ephrinB1, YFP- ephrinB2, GFP-tagged TrkB.T1 receptor (GFPTrkB.T1), or a membrane-tagged (myristoylated) form of YFP (memYFP). Time-lapse microscopy was done as above. Each movie was analyzed for trans-endocytosis events and counted as positive (yes), negative (no), or ambiguous (maybe). Movies were counted as positive when a fluorescent cluster moved away from a dendrite or dendritic filopodium, or as negative when a fluorescent cluster did not move at all or moved back and forth or alongside dendrites (data not shown). The numbers of time-lapse recordings used for the analysis are indicated in the bars. Clusters covered an area of $4.9 \pm 2.8$ pixels $^{2}$ (mean \pm SD) corresponding to $1.1 \pm 0.6 \mu \mathrm{m}^{2}$. 

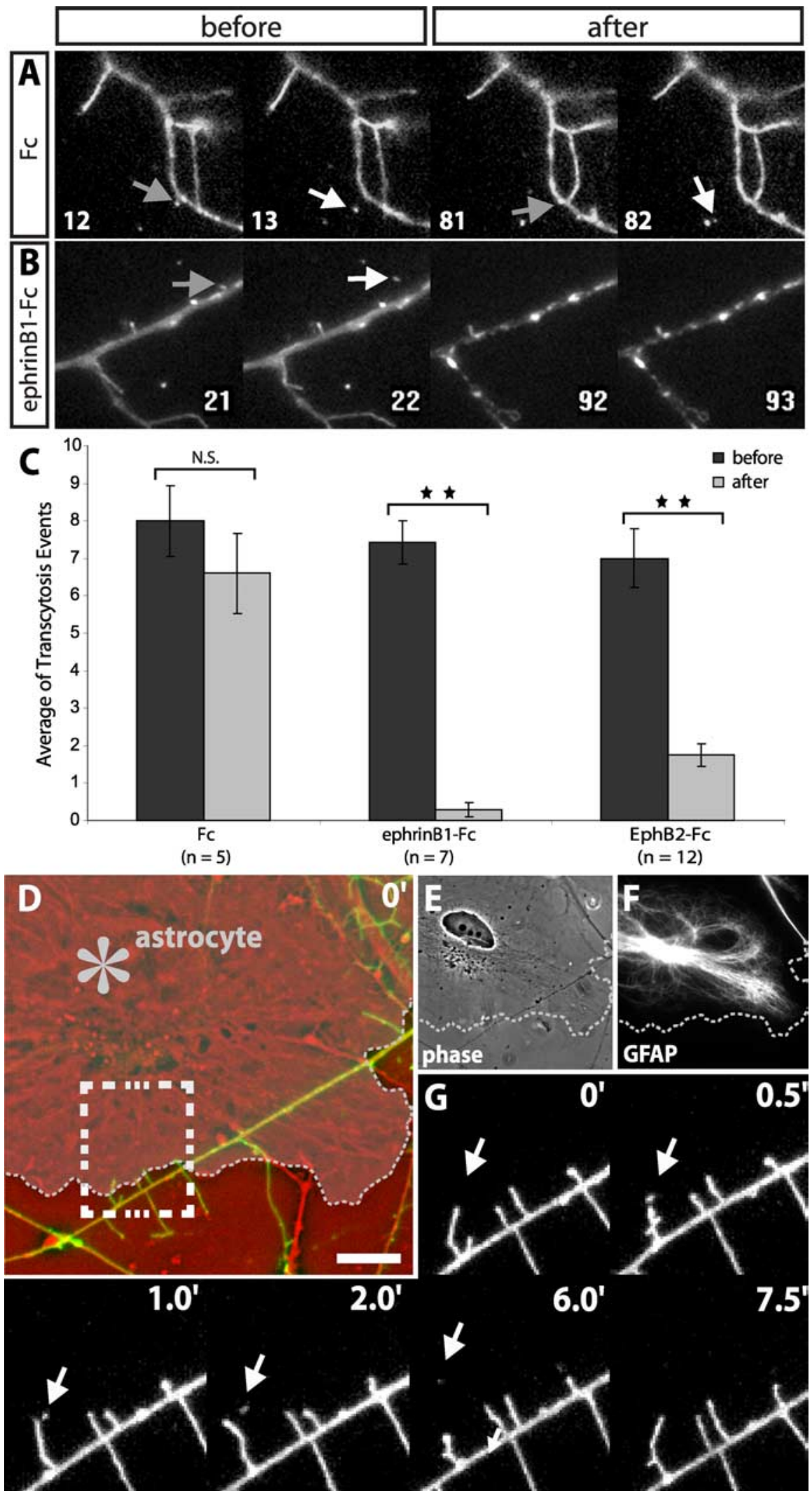

Figure 3. Inhibition of EphB2-YFP release by soluble Fc proteins and implication of astrocytes. $\boldsymbol{A}-\mathbf{G}$, Hippocampal cultures were established and imaged as in Figure 2. $\boldsymbol{A}-\boldsymbol{C}$, After 35 min of imaging, unclustered Fc, ephrinB1-Fc, or EphB2-Fc were applied to the cultures. Representative frames of the analyzed movies before and after $\mathrm{Fc}$ protein $(\boldsymbol{A})$ or ephrinB1-Fc $(\boldsymbol{B})$ application. Time points in minutes are indicated in the bottom corners. Gray arrows indicate the position at which trans-endocytosis events are just about to happen, and white arrows indicate released EphB2 clusters. $\boldsymbol{A}$, Supplemental movie S3 (available at www.jneurosci.org as supplemental material); $\boldsymbol{B}$, supplemental movie $\mathbf{4}$ (available at www.jneurosci.org as supplemental material). $\boldsymbol{C}$, Quantification of trans-endocytosis events before and after bath application of the indicated proteins. Data are expressed as mean \pm SEM fivefold between the stimuli (e.g., between EphB2-Fc and EphB3-Fc on neurons), the sizes of clusters did not vary as much (Fig. $1 G-I)$.

\section{Release of EphB2-containing vesicles} from hippocampal neurons

To investigate whether EphB2 receptors were taken up by cells contacting neurons, we transfected an expression plasmid encoding an EphB2-YFP fusion protein (Zimmer et al., 2003) into 13-15 DIV hippocampal neurons. Expression levels of exogenous EphB2 were modestly higher compared with endogenous EphBs (supplemental Fig. 4, available at www. jneurosci.org as supplemental material). Two days later, transfected neurons in cultures containing glial cells often displayed filopodia-like protrusions at the tips of growth cones and along neurite shafts. These protrusions appeared to form contacts with other cells of the culture. During time-lapse imaging, we often observed small fluorescent clusters of EphB2-YFP at the tips of such protrusions. Occasionally, fluorescent clusters were released from the neuron or pinched off by another cell and transported away from the filopodia of the transfected neuron (Fig. 2A) (supplemental movie S1, available at www. jneurosci.org as supplemental material). Because the YFP tag was inserted into the cytoplasmic domain of EphB2, the presence of fluorescent clusters in neighboring cells indicated that the entire EphB2 molecule (including parts of the plasma membrane) had been transported.

During the release, which happened in a timescale of a few minutes, filopodia were highly mobile, possibly attributable to pulling forces executed by the engulfing cell. Small protrusions that did not undergo release remained rather motionless (supplemental movies S1, S5, available at www.jneurosci.org as supplemental material). Occasionally, the release of the EphB2-YFP cluster resulted in the shrink-

$\leftarrow$

(N.S., not significant; ${ }^{* *} p<0.0001$, Student's $t$ test). $D-G$, Association of a GFAP-positive astrocyte with pinched-off EphB2 clusters in low-density cultures. $\boldsymbol{D}$, Merge of the phasecontrast image (red) and the YFP fluorescence (green) (similar to Fig. 2). Scale bar, $10 \mu \mathrm{m}$. $\boldsymbol{E}$, Bright-field image of the cell depicted in $\boldsymbol{D}$. $\boldsymbol{F}$, The same cell immunostained with anti-GFAP antibodies (GFAP). Dashed lines in the images outline the astrocyte. G, Fluorescent time-lapse images depicting a filopodium-like protrusion (arrow) growing on top of the glial cell and showing the detachment of a fluorescent cluster. Time points of imaging are indicated in the top right corners (supplemental movie S5, available at www.jneurosci.org as supplemental material). 

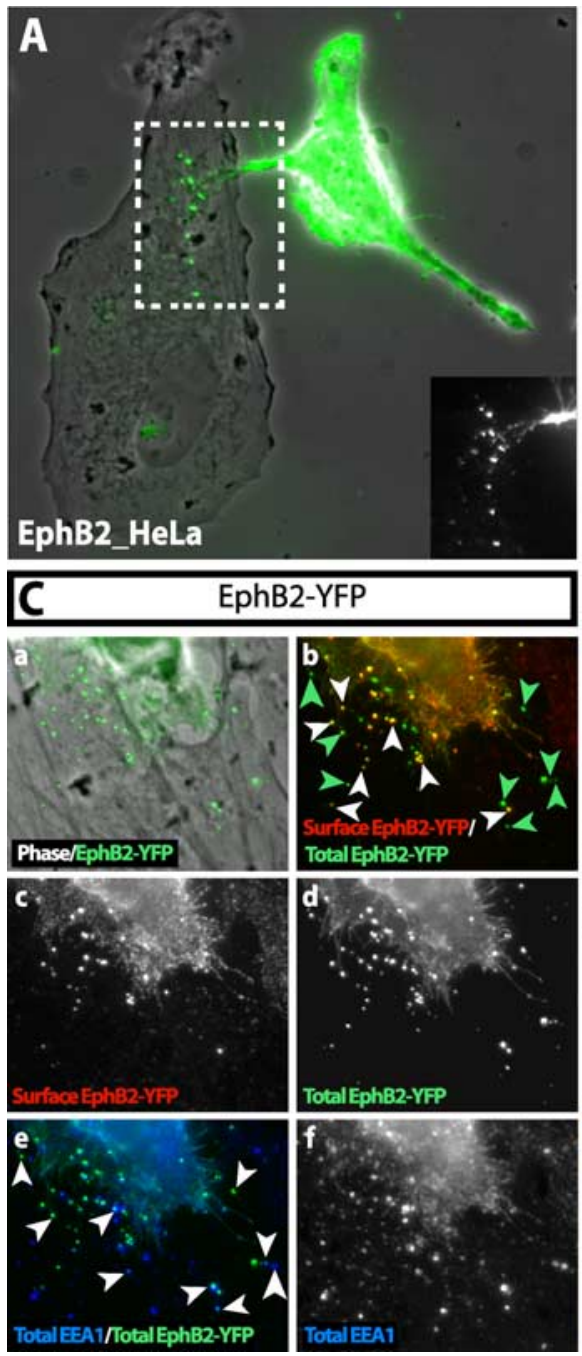
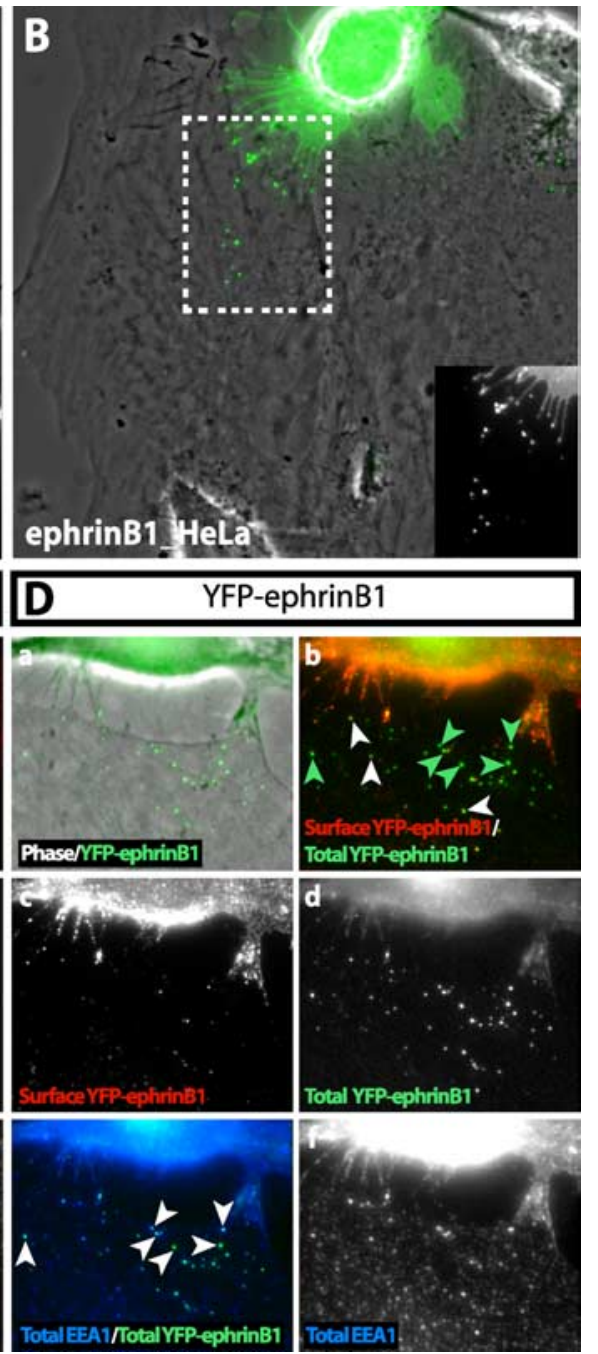

Figure 4. EphB2 and ephrinB clusters are endocytosed into astrocytes at sites of cell-to-cell contact. $\boldsymbol{A}, \boldsymbol{B}$, Mature recipient astrocytes were cultured on glass coverslips and stimulated with HeLa cells (green) transiently transfected to express either EphB2-YFP $(\boldsymbol{A})$ or YFP-ephrinB1 $(\boldsymbol{B})$. After $1 \mathrm{~h}$, cells were fixed and imaged for YFP fluorescence. Note the green fluorescent puncta in the cytosol of the astrocyte that is in contact with EphB2 $(\boldsymbol{A})$ or ephrinB1 $(\boldsymbol{B})$-expressing HeLa cells. The insets depict YFP fluorescence only. C, D, Parallel astrocyte/HeLa cultures were immunostained for EphB2 (anti-FLAG) or ephrinB1 (anti-HA) on the surface and for the endocytic marker EEA1 after the cells had been permeabilized (supplemental Materials and Methods, available at www.jneurosci.org as supplemental material). Note the presence of EphB2-positive puncta inside the astrocyte (green arrows in (b) colocalizing with EEA1 (white arrows in (e)

age and complete disappearance of filopodia (Fig. 2B) (supplemental movies S2, S5, available at www.jneurosci.org as supplemental material). Analysis of a large number of time-lapse movies revealed that, in the majority of cases, EphB2-YFP clusters were being released from the neuron (Fig. 2C). In contrast, ectopic expression of YFP targeted to the inner leaflet of the plasma membrane (memYFP) rarely triggered such a response (1 per 18 movies) (Fig. 2C). Our interpretation of these events is that the EphB2-expressing neuron is contacted by a nearby cell (most likely a glial cell; see below) that expresses ephrinBs; this contact triggers bidirectional signaling events, resulting in the uptake of the EphB2-containing vesicles by the opposing cell possibly by trans-endocytosis (Zimmer et al., 2003).

\section{Release of fluorescent clusters requires neuronal expression of EphB2}

Because cultured astrocytes express both ephrinBs and EphBs, we next reversed the experiment and asked whether exogenous ex- pression of ephrinBs in neurons would also trigger release events. We transfected neuronal cultures with expression constructs encoding fluorescently tagged ephrinB1 or ephrinB2 (YFP-ephrinB1/B2). This N-terminal modification does not interfere with receptor binding or transendocytosis (Bruckner et al., 1999; Zimmer et al., 2003). Surprisingly, in only 1 of 15 cases, ephrinBs were released toward opposing cells in the culture (Fig. 2C), indicating that the Eph/ephrin system was not equally active in both directions. This suggested that the EphB2-expressing cell was more efficient in releasing clusters than the ephrinB-expressing cell or that the pulling force exerted by the ephrinBexpressing cell was stronger than the EphB-expressing cells. We next asked whether the presence of an unrelated transmembrane protein, a GFP-tagged natural splice variant of the TrkB neurotrophin receptor (GFP-TrkB.T1) (Klein et al., 1990; Bibel et al., 1999), would also initiate this process. In only 1 of 13 cases did we observe the release of a GFP cluster (Fig. 2C).

If Eph/ephrin interactions were responsible for the observed release events, we reasoned that the addition of soluble unclustered ephrinB1-Fc or EphB2-Fc fusion proteins should block this process. We imaged neuronal cultures expressing EphB2-YFP and quantified the number of released vesicles before and after addition of Fc fusion proteins (Fig. 3A-C) (supplemental movies S3, S4, available at www. jneurosci.org as supplemental material). Whereas addition of control Fc did not significantly change the number of released vesicles, addition of ephrinB1-Fc or EphB2-Fc massively blocked the release of EphB2-YFP containing vesicles. We conclude that the release of EphB2-containing vesicles from a neuron to an opposing cell is directly mediated by ephrinB/EphB interaction and not an indirect consequence of changes in surface protein content attributable to the expression of EphB2-YFP in the neuron.

\section{Glial cells elicit the release of EphB2 clusters from neurons} We suspected that glial cells were responsible for this process because highly motile glial cells were often observed in close vicinity to the neurons undergoing EphB2 release, and filopodia growing close to or on top of glial cells often displayed highly dynamic movements and release events. To unambiguously identify the cell type, we imaged mixed low-density cultures and then fixed and stained the cultures for the glial marker GFAP. In Figure 3D-G, the contact between a glial cell and an EphB2-YFPpositive neurite was imaged during time lapse. Several fluorescent clusters were pinched off and moved away from filopodiumlike protrusions toward the glia cell (Fig. 3G) (supplemental movie S5, available at www.jneurosci.org as supplemental material). In this case, the glial cell remained well spread and displayed 
highly mobile lamellipodia. In another case, we observed a fluorescent cluster attached to a glial cell lamellipodium pulling away from the neuron (data not shown). When neurons were in contact with astrocytes, in four of four cases, the EphB2 detachment process was associated with the glial cell. If neurons had no glial contact, in one of four cases, the EphB2 release was mediated by another neuron (data not shown).

\section{Astrocytes take up fluorescent EphB2 clusters}

To investigate whether the released fluorescent clusters were internalized by an endocytic route, we modified a previously developed cell-to-cell stimulation assay (Zimmer et al., 2003) consisting of a sparse monolayer of "recipient" astrocytes on glass coverslips to which we added "stimulator" HeLa cells. If we used HeLa cells that exogenously expressed EphB2-YFP, we observed an accumulation of fluorescent clusters near the site of cell-cell contact (Fig. 4A, Ca). Comparison of surface localization of EphB2-YFP (Fig. 4Cb, red label) with its intracellular distribution (Fig. 4Cb, green label) suggested that these clusters were partially endocytosed by the astrocyte (Fig. $4 C b$, green signals and green arrowheads mark endocytosed EphB2-YFP). Immunostaining for the early endocytosis marker 1 (EEA1) (Fig. 4Ce, blue label) revealed partial colocalization with the YFP signal (Fig. $4 C e$, green label), suggesting the internalization of EphB2 via an endocytic pathway (Fig. 4Ce, overlapping blue and green label, white arrowheads). We also performed the converse experiments and cocultured recipient astrocytes with stimulator HeLa cells exogenously expressing YFP-ephrinB1. Under these conditions and different from neurons expressing YFP-ephrinB1, astrocytes readily trans-endocytosed YFP (Fig. 4D). These findings indicate that naive cultured astrocytes are able to trans-endocytose EphB2-YFP from opposing cells.

\section{Discussion}

Here we have shown the release of membrane material from healthy (non-injured) neurons to cocultured glial cells. Experiments with transfected HeLa cells and primary astrocytes suggest that astrocytes trans-endocytose this membrane material. These events require the direct interaction between EphB2 on neurons and its binding partners on astrocytes (ephrinBs). Transendocytosis among hippocampal neurons and with glia has been observed previously in the intact hippocampus through serial section electron microscopy (Spacek and Harris, 2004). Doublemembrane structures called spinules were enriched in perisynaptic glia interacting directly with synapses on thin dendritic spines as opposed to mushroom spines. Our findings are consistent with a model that says that neuronal material including membranes is released from thin protrusions at sites of neuron-to-glia contact. We suggest that Eph/ephrin signaling is critically involved in this process.

What could be the general functions of trans-endocytosis from neuron to glia? Besides microglia, astrocytes have been known to phagocytose degenerating axonal boutons (Bechmann and Nitsch, 1997; Nedergaard et al., 2003; Volterra and Meldolesi, 2005). In Drosophila, glial cells are recruited to healthy neurons for development-appropriate axonal pruning (Awasaki and Ito, 2004; Watts et al., 2004). The type of trans-endocytosis observed in our mixed cultures does not resemble traditional astrocytic phagocytosis of degenerating axons. Rather, these events may be regulating structural remodeling between neuronal protrusions and glia under physiological conditions. At the mammalian neuromuscular junction, axonal inputs are eliminated under physiological conditions by a mechanism that shows some resemblance to the events described here. As axons disappear, they shed membrane-bound "axosomes" that are formed by engulfment of axon tips by glial (Schwann) cells (Bishop et al., 2004). Glia cells are active partners at CNS synapses, dynamically regulating synaptogenesis and synaptic transmission (Nedergaard et al., 2003). The observed exchange of membrane material may be part of an active information exchange that enables glial cells to regulate specific aspects of synapse formation, maintenance, and elimination.

What could be the specific function of EphB/ephrinB signaling and trans-endocytosis in perisynaptic glia? Activation of Eph/ ephrin signaling at cell-to-cell interaction sites typically elicits some type of repulsive behavior (although adhesive responses are possible, too). Neuronal progenitor cells in the segmented hindbrain sort themselves into alternating Eph- and ephrin-positive segments by avoiding contact with cells expressing their binding counterparts (Sela-Donenfeld and Wilkinson, 2005); migrating growth cones expressing Eph receptors turn away from cells that present the correct ephrin (Pasquale, 2005). Mechanistically, Eph/ephrin signaling may be involved at two separate levels: (1) it may activate the cellular machinery necessary for membrane traffic leading to (macro-) pinocytosis and the execution of the pinching-off process; (2) it may instruct the cell to retract its processes away from its neighboring cell, thereby providing some of the pulling forces for the pinching-off process. It will be important in the future to functionally separate membrane trafficking from cell retraction and to identify the molecular machinery driving these processes.

In summary, our observations add to the increasing richness in glial cell biology and support the idea that glial cells act instructively in neuronal developmental processes. Moreover, the implication of the Eph/ephrin signaling system provides an entry point into the molecular pathways that drive neuron-to-glia interactions.

\section{References}

Awasaki T, Ito K (2004) Engulfing action of glial cells is required for programmed axon pruning during Drosophila metamorphosis. Curr Biol 14:668-677.

Bechmann I, Nitsch R (1997) Astrocytes and microglial cells incorporate degenerating fibers following entorhinal lesion: a light, confocal, and electron microscopical study using a phagocytosis-dependent labeling technique. Glia 20:145-154.

Bibel M, Hoppe E, Barde YA (1999) Biochemical and functional interactions between the neurotrophin receptors trk and p75NTR. EMBO J 18:616-622.

Bishop DL, Misgeld T, Walsh MK, Gan WB, Lichtman JW (2004) Axon branch removal at developing synapses by axosome shedding. Neuron 44:651-661.

Bruckner K, Pablo Labrador J, Scheiffele P, Herb A, Seeburg PH, Klein R (1999) EphrinB ligands recruit GRIP family PDZ adaptor proteins into raft membrane microdomains. Neuron 22:511-524.

Bundesen LQ, Scheel TA, Bregman BS, Kromer LF (2003) Ephrin-B2 and EphB2 regulation of astrocyte-meningeal fibroblast interactions in response to spinal cord lesions in adult rats. J Neurosci 23:7789-7800.

Conover JC, Doetsch F, Garcia-Verdugo JM, Gale NW, Yancopoulos GD, Alvarez-Buylla A (2000) Disruption of Eph/ephrin signaling affects migration and proliferation in the adult subventricular zone. Nat Neurosci 3:1091-1097.

Davy A, Soriano P (2005) Ephrin signaling in vivo: look both ways. Dev Dyn 232:1-10

de Hoop MJ, Olkkonen VM, Ikonen E, Williamson E, von Poser C, Meyn L, Dotti CG (1994) Semliki Forest virus as a tool for protein expression in cultured rat hippocampal neurons. Gene Ther 1 [Suppl 1]:S28-S31.

Eichmann A, Makinen T, Alitalo K (2005) Neural guidance molecules regulate vascular remodeling and vessel navigation. Genes Dev 19:1013-1021.

Flanagan JG (2006) Neural map specification by gradients. Curr Opin Neurobiol 16:59-66. 
Goetze B, Grunewald B, Kiebler MA, Macchi P (2003) Coupling the ironresponsive element to GFP — an inducible system to study translation in a single living cell. Sci STKE 2003:PL12.

Goldshmit Y, Galea MP, Wise G, Bartlett PF, Turnley AM (2004) Axonal regeneration and lack of astrocytic gliosis in EphA4-deficient mice. J Neurosci 24:10064-10073.

Harbott LK, Nobes CD (2005) A key role for Abl family kinases in EphA receptor-mediated growth cone collapse. Mol Cell Neurosci 30:1-11.

Henderson JT, Georgiou J, Jia Z, Robertson J, Elowe S, Roder JC, Pawson T (2001) The receptor tyrosine kinase EphB2 regulates NMDA-dependent synaptic function. Neuron 32:1041-1056.

Klein R, Conway D, Parada LF, Barbacid M (1990) The trkB tyrosine protein kinase gene codes for a second neurogenic receptor that lacks the catalytic kinase domain. Cell 61:647-656.

Kullander K, Klein R (2002) Mechanisms and functions of Eph and ephrin signalling. Nat Rev Mol Cell Biol 3:475-486.

Marston DJ, Dickinson S, Nobes CD (2003) Rac-dependent transendocytosis of ephrinBs regulates Eph-ephrin contact repulsion. Nat Cell Biol 5:879-888.

Murai KK, Nguyen LN, Irie F, Yamaguchi Y, Pasquale EB (2003) Control of hippocampal dendritic spine morphology through ephrin-A3/EphA4 signaling. Nat Neurosci 6:153-160.

Nedergaard M, Ransom B, Goldman SA (2003) New roles for astrocytes: redefining the functional architecture of the brain. Trends Neurosci 26:523-530.

Palmer A, Klein R (2003) Multiple roles of ephrins in morphogenesis, neuronal networking, and brain function. Genes Dev 17:1429-1450.

Pasquale EB (2005) Eph receptor signalling casts a wide net on cell behaviour. Nat Rev Mol Cell Biol 6:462-475.

Sela-Donenfeld D, Wilkinson DG (2005) Eph receptors: two ways to sharpen boundaries. Curr Biol 15:R210-R212.

Spacek J, Harris KM (2004) Trans-endocytosis via spinules in adult rat hippocampus. J Neurosci 24:4233-4241.

Volterra A, Meldolesi J (2005) Astrocytes, from brain glue to communication elements: the revolution continues. Nat Rev Neurosci 6:626-640.

Wang Y, Ying GX, Liu X, Wang WY, Dong JH, Ni ZM, Zhou CF (2005) Induction of ephrin-B1 and EphB receptors during denervation-induced plasticity in the adult mouse hippocampus. Eur J Neurosci 21:2336-2346.

Watts RJ, Schuldiner O, Perrino J, Larsen C, Luo L (2004) Glia engulf degenerating axons during developmental axon pruning. Curr Biol 14:678-684.

Wilkinson DG (2003) How attraction turns to repulsion. Nat Cell Biol 5:851-853.

Zimmer M, Palmer A, Kohler J, Klein R (2003) EphB-ephrinB bidirectional endocytosis terminates adhesion allowing contact mediated repulsion. Nat Cell Biol 5:869-878. 